\title{
Riesgo y percepción en el desarrollo de la imagen turística de Brasil ante los mega-eventos deportivos
}

\author{
Jaime Álvarez de la Torre* \\ Universidad de A Coruña, España \\ Diego Rodríguez-Toubes Muñiz** \\ Universidad de Vigo, España
}

\begin{abstract}
Resumen: En el imaginario occidental existen una serie de atributos a la hora de referirse a Brasil, unos de componente positivo como el valor tropical, la alegría y lo exótico, y otros con un componente negativo como la inseguridad, la desigualdad o marginalidad. Ante la próxima celebración de dos mega-eventos deportivos como son el Mundial de Fútbol 2014 y los Juegos Olímpicos 2016, Brasil se encuentra en un lugar intermedio entre el estereotipo y la realidad. La adecuación y preparación del país a las necesidades de estos eventos puede ser el medio para mitigar ciertos estereotipos y riesgos, y avanzar en un desarrollo sostenible del turismo con vocación al largo plazo, integrando a la comunidad residente en los planes de seguridad. En este trabajo se realiza una revisión sobre el impacto de la imagen y el concepto de seguridad en la elección de viaje a un destino concreto. Se valora la experiencia de visita al destino como medio para reducir los estereotipos haciendo que la imagen tienda a ser más realista, compleja y diferenciada, potenciada por una correcta gestión de la información.
\end{abstract}

Palabras clave: Brasil, imagen turística, mega-eventos, percepción de riesgo, seguridad

Risk and perception in the development of Brazil's tourist image facing the sports mega-events

Abstract: There are a number of attributes in the Western world when referring to Brazil that are stereotypes, some have a positive component as "tropical value", "joy" and "exotic", and others have a negative component as "insecurity"," inequality" or "marginalization ". With the upcoming celebration of two sporting massive events such as the 2014 World Cup and 2016 Olympics, Brazil is located between the stereotype and reality. The suitability and readiness of the country to the requirements of these events can be the way to mitigate certain stereotypes and risks, and make progress in the sustainable development of tourism in the long term, integrating also the local community in the government's security plans. In this paper we review the impact of the image and safety in the travel decision. Previous experience of visiting the destination has the virtue of reducing the negative stereotypes; this makes the image more realistic, complex and differentiated, enhanced by a proper information management.

Keywords: Brasil, mega-events, risk perception, safety, tourist image

* Universidad de A Coruña, España. E-mail: jaime.delatorre@udc.es

** Universidad de Vigo. Organización de empresas y marketing. E-mail: drtoubes@uvigo.es 


\section{Introducción}

Los retos a los que se enfrenta Brasil con la organización de un Mundial de Fútbol y los Juegos Olímpicos (JJOO) en un período de tan sólo dos años, suponen una auténtica prueba de fuego de cara a la exposición internacional del país. La apuesta por los grandes eventos, y por el turismo de negocios en general, constituye una de las líneas estratégicas por parte de las instituciones brasileñas. Ante la proximidad de dichos eventos parecen empezar a surgir dudas acerca de si la estrategia de acondicionamiento del país de cara a los mega-eventos no será más que un espejismo fugaz o por el contrario tendrá carácter de permanencia.

Unas Olimpiadas llevan intrínsecos una serie de atributos que hacen que el destino proyecte una imagen positiva a través de valores como la globalidad, dignidad, modernidad, multiculturalidad y dinamismo. Se trata de un evento con más de 3.000 años de historia y con un significado que trasciende más allá de lo meramente deportivo, con elementos intangibles más difíciles de cuantificar y medir, pero al mismo tiempo con una vertiente eminentemente económica. Se conecta de este modo dos realidades bien diferentes aunque no independientes: la visión económica y la visión "olímpica tradicional". Payne (2007) califica de paradoja la relación entre ambos conceptos, ya que realmente son los valores no comerciales los que contribuyen a aportar el gran valor comercial -económico- que tiene el evento que, al fin y al cabo, sigue siendo el indicador primordial a la hora de medir el impacto del acontecimiento.

El establecimiento de estereotipos es una herramienta utilizada para homogeneizar la imagen que se tiene de cierta colectividad entendida como un grupo, una ciudad, país, región o continente. La imagen de inseguridad está presente como estereotipo negativo y es uno de los elementos generadores de la percepción de riesgo del destino Brasil. Existen, desde luego, otros atributos, pero el factor de inseguridad, agravado por la imagen difundida en la prensa internacional o las recomendaciones de viaje oficiales emitidas por las oficinas de asuntos exteriores de los países emisores, es una variable muy influyente en la toma de decisión de viaje del turista. Para Brasil surge una oportunidad única de enfocar y promocionar la imagen exterior del país en el exterior, para ello hay es conveniente comprender los postulados de la teoría de percepción del riesgo al establecimiento de la imagen y promoción exterior de Brasil ante los próximos mega-eventos.

\section{Repercusión de los mega-eventos y per- cepción de riesgo}

Una herramienta empleada por países emergentes o países en vías de desarrollo como medio para posicionarse de modo adecuado en el panorama internacional es lanzarse en la aventura de organizar eventos deportivos globales (Cornelissen, 2004). Los mundiales de Corea 2002 ó Sudáfrica 2010 son ejemplos de aprovechamiento y regeneración del país a todos los niveles de cara a una apertura al exterior. La investigación sobre las repercusiones y efectos de este tipo de eventos en los destinos sede ha ido creciendo continuamente, sin duda por la importancia que supone para los países de acogida a todos los niveles socioeconómicos. Ritchie (1984) entiende el concepto de hallmark como aquellos eventos que se desarrollan, en una única edición o repetidos en el tiempo, para mejorar el conocimiento, atractivo y rentabilidad de un destino turístico en el corto y/o largo plazo. Los JJOO o el mundial de fútbol constituyen los auténticos hallmarks en cuanto a eventos, constituyendo verdaderos referentes por importancia, seguimiento e impacto. Un estudio realizado a turistas que acudieron al mundial de Corea 2002 concluyó que la imagen del país había mejorado con respecto a antes del evento así como la percepción de inseguridad había decrecido notablemente (Kim y Morrison, 2005). Experiencias anteriores en otras sedes demuestran cómo puede modificarse la imagen del país en el extranjero; durante el Mundial de fútbol de Alemania 2006, la campaña A time to make friends se fundamentó en la necesidad de cambiar la imagen que se tenía del pueblo alemán como poco simpático y con pocas demostraciones de sensibilidad, atención y cordialidad.

Es común que una de las mayores preocupaciones sea la rentabilidad económica tras la enorme inversión que estos eventos suele acarrear. Uno de los mayores esfuerzos presupuestarios, y ejes de actuación, lo constituye el gasto en medidas de seguridad, justificado en parte por la creciente amenaza global que existe hoy en día en cualquier punto del planeta. A pesar de todo, puede haber turistas que aun juzgando un destino como peligroso decidan visitarlo igual (Larssen, 2009). En unos JJOO o un mundial la percepción del riesgo difiere según a qué tipología turística nos estemos refiriendo y a las propias características del visitante. A través de algunos estudios ha podido constatarse que el turista extranjero valora un mayor abanico de riesgos, pero igualmente, su mayor motivación para viajar reduce ese sentimiento de ansiedad 
(Reisinger y Mavondo, 2005). En las Olimpiadas de Londres 2012 la percepción sobre el riesgo de los visitantes estadounidenses a Londres fue baja, quizá por el hecho de desplazarse a una capital cosmopolita y metropolitana de una sociedad avanzada que consideran similar a la propia (Schoreder, Penninngton-Gray, Kaplanidou y Zhan, 2013).

Puede añadirse el hecho de que, aun asumiendo cierto tipo de riesgos, cuando un individuo quiere ser espectador de este tipo de eventos, no tiene la posibilidad de escoger otro destino más seguro (Neirotti y Hilliard, 2006). Aunar bajo un mismo evento ocio, deporte y turismo puede provocar una disminución en la percepción de dicho riesgo (Leep y Gibson, 2003). Incluso es socialmente aceptado que cierto tipo de eventos lleven una serie de riesgos inherentes, y es que la lógica parece indicar que si el delito es una figura que siempre ha existido, la aglomeración de personas en ciertos espacios propicia la aparición de figuras delictivas (Jennings y Lodge, 2009).

En una actividad como el turismo, dónde la experiencia y las expectativas del viajero representan un papel esencial, la idea de riesgo y su percepción son un aspecto clave a tener en cuenta a la hora de establecer las estrategias de planificación de la imagen turística de un destino. La consideración del riesgo es susceptible de condicionar al potencial visitante en el momento de la toma de decisión. En el caso de los eventos programados en Brasil, sin embargo, existe un factor que atenúa dicha consideración de riesgo, ya que ante unos eventos de este calibre la influencia en la percepción del riesgo es menor al ser acontecimientos puntuales y excepcionales a los que el visitante es posible que no tenga muchas más oportunidades de acudir. En este caso el riesgo implica asumir ciertas amenazas, pero con la contrapartida de obtener un beneficio, en términos de satisfacción o cumplimiento de expectativas.

En la identificación del componente de riesgo Korstanje (2010) relaciona el riesgo con la probabilidad de sufrir daños, parciales o totales, o con consecuencias negativas inesperadas. En esta relación se pueden apreciar dos elementos de gran importancia como la vulnerabilidad turística y el grado de satisfacción (figura 1).

La relación crimen-percepción de riesgo suele ser de las más influyentes en la conducta del visitante (Barker, Page y Meyer, 2003; George, 2010) en tanto que es un riesgo que afecta directamente a su integridad. La población puede aceptar o asumir otro tipo de riesgos como los climáticos con mayor tolerancia o establecer otro
Figura 1. Repercusiones de consideración del riesgo

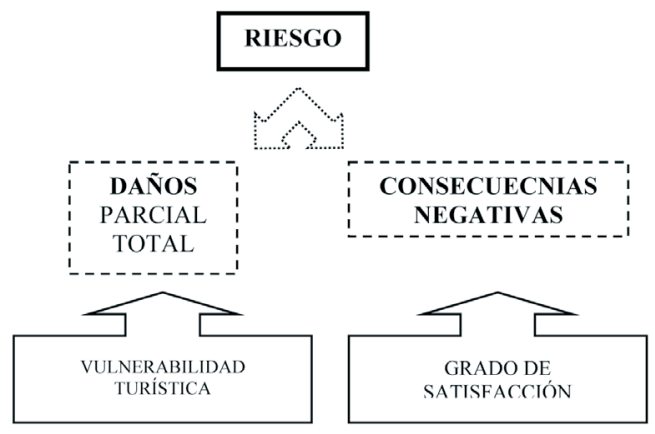

Fuente: Elaboración propia a partir de Korstanje (2010)

tipo de medidas de prevención. En el caso de la seguridad personal y criminalidad, estas medidas de prevención deben venir por parte de las administraciones públicas nacionales y a su vez, ser capaces de implicar al resto de estamentos y clases de la sociedad. La figura del turista es más vulnerable y proclive a padecer ciertos acontecimientos negativos convirtiéndose en presa fácil para la delincuencia común y el crimen organizado (Brás y Rodrigues, 2010; Leep y Gibson, 2003; Stangeland, Díez y Durán, 1998), además al referirse a mega-eventos deportivos, es previsible que el turista haga ostentación pública de su condición y origen en aras de apoyar a sus representantes en las competiciones y mostrar su orgullo nacional, pudiendo llegar a ser la causa del problema.

\section{Estereotipos e influencia mediática en la imagen de Brasil}

La proliferación de estudios de imagen empieza a ser una disciplina cada vez más desarrollada dentro de la investigación turística. Constituye un campo científico muy fértil para delimitar las motivaciones, percepciones 0 conocimientos a la hora de conocer qué factores influyen en la imagen de un destino y en el caso que nos ocupa, el grado de penetración y resistencia de ciertos estereotipos establecidos en el imaginario colectivo.

En líneas generales la utilización de estereotipos en cuanto a nacionalidades se refiere, es un modo de representar a una comunidad heterogénea y compleja de un modo homogéneo. Es el modo en cómo otros ven a cierta colectividad; pudiéndonos referir a una familia, a un barrio, ciudad, país, región o incluso continente. El estereotipo a pesar de ser un concepto amoldable a 
diversas circunstancias y contextos, suele tener cierto grado de resistencia sobre todo si conlleva carácter negativo. La trascendencia y causalidad que puede tener cierto suceso no será la misma según a que país nos estemos refiriendo y según el origen y características del emisor y receptor de dicho mensaje.

Ribeiro (2002) califica como "tropicalismo" uno de los principales estereotipos de Brasil. Sin entrar en detalles geográficos o antropológicos, esta visión exótica es fruto de la propia historia del país y de su diversidad étnica y territorial, y por tanto dentro del imaginario occidental suele ser uno de los referentes. Pero a su vez, el autor advierte que a pesar de ser el tropicalismo un concepto con cierta fundamentación, puede abordarse como aspecto positivo o negativo. Dentro del primero se enmarcarían todos los recursos que suelen ser promocionados por el propio sector turístico como la alegría de sus gentes, el exotismo, las playas, carnaval, etc.; pero igualmente el carácter tropical, también encierra la idea de peligrosidad, riesgo, delincuencia o incluso cierta dosis machista en relación entre sexos. Esta seña de identidad abarca todos los ámbitos de Brasil; desde el tratamiento mediático, hasta la promoción o uso institucional. De hecho en la elaboración de la marca Brasil se hace referencia precisamente a la exuberancia, mezcla de razas, mestizaje, hospitalidad o alegría. Esta imagen del país constituye uno de los principales activos de promoción turística y, ante los próximos eventos, seguirá siendo el hilo conductor de la política promocional.

La organización de un mega-evento deportivo puede ser utilizada como arma política en tanto que es un modo de exponer ideas de apertura, fomento de empleo, calidad de vida y retorno de inversiones que repercutirá en toda la sociedad (Radicchi, 2012). Bajo esta visión del mega-evento como instrumento político, el tratamiento mediático que se le haga, afectará a su desarrollo, y por ende, al turismo. Si ya de por sí los medios de comunicación pueden ser oportunistas, parciales y subjetivos; como elementos formadores de imagen de un destino adquieren mayor relevancia.

El utilizar los medios de comunicación como fuente de información para crear una imagen de destino puede sesgar bastante la idea que un individuo pueda hacerse de un país. A menudo la presentación mediática sobre cierto acontecimiento o idea difiere bastante de la realidad por el tratamiento (interesado o no) que se le da. Este hecho puede provocar que a la hora de la elección del destino o de formación de la imagen, se produzca una discriminación de ciertos valo- res, recursos y atributos en beneficio de ciertos tópicos y estereotipos.

La falta de experiencia previa directa a un destino fuerza al turista a emplear otro tipo de fuentes de información sobre el destino como son los medios de comunicación. En los principales mercados emisores, el tratamiento de ciertos atributos o sucesos del destino turístico pueden tratados con excesivo sensacionalismo o llegar a ser manipulados con fines políticos. Un estudio sobre el tratamiento mediático de la inmigración brasileña en España ${ }^{1}$, desveló que gran parte de las informaciones centradas en este colectivo recibían un tratamiento negativo al estar asociadas con temáticas como prostitución, delincuencia, violencia de género o tráfico de drogas. La falta de contraste de este tipo de noticias refuerza que los estereotipos negativos sigan creciendo e influenciando en la formación de la imagen de Brasil. Hacer hincapié y relacionar constantemente delincuencia o prostitución con una nacionalidad determinada, no resulta beneficioso para un destino que quiere postularse como motor de Sudamérica y en el que se van a celebrar con tan sólo dos años de separación los eventos deportivos con más repercusión a nivel mundial.

Estas ideas surgen del tratamiento mediático de diversas experiencias pasadas; la presentación de imágenes para dar cobertura a ciertas noticias sobre seguridad, prostitución o drogas refuerzan la idea de país en desarrollo; y por otro lado, la utilización de imágenes arquetípicas de destinos como Río de Janeiro para la promoción turística, refuerzan la idea "tropicalista" de Brasil (Ribeiro, 2002). Para Soto (2005) en muchas ocasiones el tratamiento mediático no se corresponde con la auténtica realidad del asunto. Sin embargo, noticias de sucesos delictivos concretos ${ }^{2}$, problemas de inseguridad o desórdenes públicos en el destino, incrementan el nivel de preocupación e incertidumbre del turista potencial.

Si seguimos los planteamientos de Gartner (1994) cuando aplica los planteamientos de formación de la imagen de Gunn (1988) para el caso de Brasil, podemos constatar el peso preponderante de lo que califica como fuentes de información inducidas y orgánicas en la formación de la imagen antes de la visita. En el año 2011 las principales fuentes de información para los visitantes fueron Internet -inducida- $(32,6 \%)$ y amigos y familiares -orgánica- $(28,5 \%)$. En un intervalo corto de tiempo, las fuentes de información inducidas abiertas han sobrepasado a las fuentes orgánicas, sin duda por su rápida evolución. Hoy en día casi todos los medios de comunicación se sirven de las nuevas TIC's 
como soporte de difusión por su elevado grado de amplitud y penetración, pero además también se les suele dotar de cierto grado de credibilidad (Santana y Palacio, 2004). Saber gestionar la información es un punto clave para gestionar la incertidumbre que, en último término, es la que condiciona la percepción de riesgo. Por ello, para Slevitch y Sharma (2008: 87) "gestión de riesgo es, en gran medida, gestión de la información”.

\section{El problema de la inseguridad en Brasil}

En los últimos cuatro años Brasil ha experimentado una tendencia creciente en las llegadas del turismo internacional (figura 2). La celebración de los mega-eventos proporcionará un importante impulso al número de llegadas, y ya en el 2013 Brasil se expondrá a un test que medirá su capacidad y preparación para los mega-eventos de 2014 y 2016 con la celebración de la Copa Confederaciones de Fútbol (antesala del Mundial) y la Jornada Mundial de la Juventud (a la que se calcula que acudirán más de dos millones de participantes).

Aunque a priori se tenga asegurado un incremento importante en la cifra de llegadas, se debe prestar la debida atención a los estereotipos existentes en el imaginario occidental que, como se tratado anteriormente, identifica la inseguridad como uno de ellos. En el estado de Río de Janeiro, los datos oficiales sobre lo que se califican como "crímenes violentos", que a priori más afectarían a los turistas, se han visto reducidos en los últimos tiempos. Comparando los años 2010 y 2011, los homicidios dolosos se han reducido en un $10,2 \%$, los robos en transporte público en un $21,2 \%$ y a transeúntes en un $13,2 \%$. El único incremento es en agresiones sexuales, en un $6,1 \%$, si bien es cierto que motivado por reformas en materia penal que amplían las acciones punibles bajo este tipo delictivo (Instituto de Seguridad Pública, 2012).

Figura 2. Llegadas de turismo internacional a Brasil

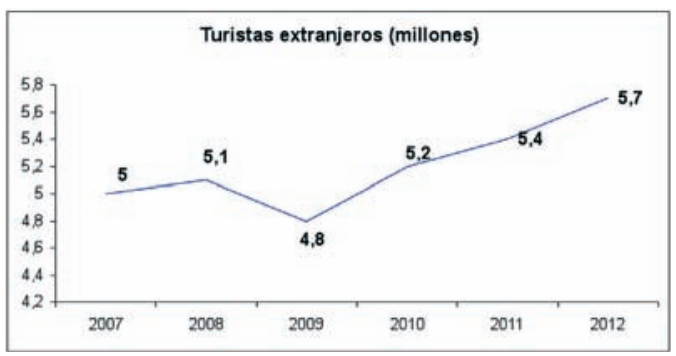

Fuente: Ministerio de Turismo de Brasil (2012)
En el caso brasileño las amenazas reales y propias del destino pueden tener mayor influencia sobre los turistas en el momento de la decisión de viaje debido a la existencia de ciertas ideas arquetípicas en el imaginario del visitante.

La planificación turística de Brasil en el periodo 2010-214 también reconoce la inseguridad como una de las debilidades a superar, preferentemente en los barrios de las ciudades sede de los eventos. Además, se señala la falta de una cultura de viaje en el ciudadano brasileño que, en general, concibe el turismo como un producto de élite o reservado a extranjeros (Ministerio de Turismo de Brasil, 2010). Esta actitud conduce a los llamados "efectos no deseados del turismo", efectos que se ven agravados en caso de los mega-eventos, y que podrían derivar en un choque cultural entre sociedad receptora y visitantes. Esta situación provocaría que los visitantes precisaran de sobreprotección convirtiendo los destinos en burbujas, reflejando una imagen alejada de la imagen de hospitalidad y alegría proyectada. La regeneración urbana requerida en muchas zonas de favelas está derivando en expropiaciones que en algunos casos no están siendo acogidas satisfactoriamente por la población local. A medida que se acercan las fechas de celebración de los mega-eventos, parece estar surgiendo una ola de escepticismo a todos los niveles en el modo en cómo se está planificando el territorio. Lo que en principio se promociona como una oportunidad de mejora e impulso para el país, puede acabar derivando en un creciente pesimismo y escepticismo provocando cierta conducta de rechazo frente al turismo de mega eventos.

En los visitantes, el nivel de preocupación puede verse incrementado a la hora de valorar las opciones de elección de destino turístico, entendiendo la preocupación como un ejercicio mental relativo al futuro y a sus consecuencias (Borkovec, 1994). La percepción de seguridad es personal y difícil de catalogar. Se trata, al fin y al cabo, de un modo personal del individuo de minimizar, prevenir o relativizar cierto tipo de amenazas o riesgos, y difiere según nos estemos refiriendo a residentes o visitantes. El residente que convive con este problema puede ser más crítico y exigente con la seguridad que el visitante, quizá más conformista o sobreprotegido por el sistema en detrimento de la población local que sólo ve esfuerzos en materia de seguridad para con el turista (Amorín, Gándara, Tarlow y Korstanje, 2012).

Si se acepta la existencia del estereotipo de inseguridad resulta interesante valorar el grado de satisfacción y la valoración de seguridad por 
Tabla 1. Valoración de la seguridad en Brasil del turista extranjero

\begin{tabular}{|l|c|c|c|c|c|c|}
\hline \multirow{2}{*}{$\begin{array}{c}\text { País } \\
\text { procedencia }\end{array}$} & $\begin{array}{c}20 \\
\text { Nivel alto } \\
\text { Satisfacción }\end{array}$ & $\begin{array}{c}\text { Valoración } \\
\text { positiva de } \\
\text { seguridad }\end{array}$ & $\begin{array}{c}\text { Nivel alto } \\
\text { Satisfacción }\end{array}$ & $\begin{array}{c}\text { Valoración } \\
\text { positiva de } \\
\text { seguridad }\end{array}$ & $\begin{array}{c}\text { 2010 } \\
\text { Nivel alto } \\
\text { satisfacción }\end{array}$ & $\begin{array}{c}\text { Valoración } \\
\text { positiva de } \\
\text { Seguridad }\end{array}$ \\
\hline Alemania & $85,5 \%$ & $69,8 \%$ & $83,3 \%$ & $74,9 \%$ & $76,3 \%$ & $75,2 \%$ \\
\hline Argentina & $91,7 \%$ & $92,8 \%$ & $93,7 \%$ & $93,6 \%$ & $92,1 \%$ & $93 \%$ \\
\hline Chile & $89,5 \%$ & $86,3 \%$ & $86,7 \%$ & $87,5 \%$ & $85,5 \%$ & $87,9 \%$ \\
\hline España & $84,3 \%$ & $71,3 \%$ & $76,9 \%$ & $68,4 \%$ & $7,3 \%$ & $72,7 \%$ \\
\hline EEUU & $86,5 \%$ & $74,2 \%$ & $80,7 \%$ & $73,4 \%$ & $76,7 \%$ & $75,5 \%$ \\
\hline Francia & $86,3 \%$ & $76,9 \%$ & $81,2 \%$ & $80 \%$ & $79,8 \%$ & $79,2 \%$ \\
\hline Inglaterra & $81,3 \%$ & $71,6 \%$ & $78,2 \%$ & $73,3 \%$ & $78 \%$ & $76,1 \%$ \\
\hline México & $87,1 \%$ & $79,9 \%$ & $82,1 \%$ & $84,8 \%$ & $83,7 \%$ & $86,6 \%$ \\
\hline
\end{tabular}

Fuente: Ministerio de Turismo Brasil (2012)

parte del turista una vez que ha realizado el viaje. En la tabla 1 se observa como la valoración de la seguridad de Brasil ha ido mejorando para los turistas de los principales mercados emisores desde 2009 al 2011. Los visitantes procedentes de países sudamericanos son los que otorgan mayor valoración a aspectos relacionados con la seguridad tras la visita a Brasil, quizá por estar más relacionados en su día a día con estos problemas y encontrarse todavía en la fase de conquista de niveles más bajos de delincuencia, mientras que los países occidentales pueden mostrarse más exigentes en estos términos.

El perfil de turista que viaja a Brasil en los últimos años es el de una persona de edad comprendida entre los 25 y los 50 años (el $68 \%$ de los casos) y al que no parece afectarle la imagen de inseguridad del país. En un $69 \%$ de los casos ya había estado en Brasil y un 95,4\% estaría dispuesto a volver (Ministerio de Turismo, 2012). El hecho de visitar un destino contribuye a la creación de una imagen más compleja, holística y única (Etchner y Ritchie, 1993 y Baloglu y Mangaloglu, 2001) y en este caso nos indica además que contribuye a reducir la influencia del estereotipo negativo. La experiencia con el destino provoca un cambio de imagen al ser más realista y personal (Gartner, 1994).

\section{Conclusiones}

El abanico de riesgos que afectan al turismo es muy variado y es evidente que condiciona la elección de las sedes de unos JJOO o el Mundial de fútbol. La elección de una sede para estos eventos es un proceso largo, costoso y no falto de interferencias de cualquier tipo. Tanto el COI como la FIFA como entidades organizadoras señalan la capacidad del deporte como vehículo hacia la paz y entendimiento entre naciones, en este sentido procuran que olimpiadas y mundiales de fútbol se celebren alternamente en los diversos continentes como símbolo de globalidad y unión entre pueblos. Además, existen una serie de factores políticos, sociales y económicos que acaban siendo los decisivos en el proceso de decisión.

Brasil debe realizar un esfuerzo en gestionar ciertos conflictos político-sociales como la delincuencia, la marginalidad y la diferencia de clases entre su población. A medida que se acerca la fecha de dichos eventos, parecen surgir voces discordantes que ponen en tela de juicio los verdaderos beneficios para la población local. Existe cierto escepticismo sobre si realmente la pacificación y regeneración de territorios es un proceso de doble sentido que englobe a turistas con visitantes. Actualmente el riesgo en Brasil sobre la integridad del turista es algo real, y algunas voces han defendido que la labor de las entidades gobernantes y responsables del turismo y la seguridad no se debe limitar exclusivamente a "sobreproteger" al turista, como si el país entero fuera un gran resort, sino también, y principalmente, a implicar a la comunidad local en la acogida del turista.

Los mega-eventos que acogerá Brasil en los próximos años tienen que ser un medio más que permita potenciar la imagen de marca del país como destino turístico. Aunque ser sede 
de cualquier evento de estas magnitudes es el mejor trampolín para favorecer la exposición mundial de un territorio, estos acontecimientos no dejan de ser hechos puntuales y limitados en el tiempo. La creación de una imagen amoldada forzosamente a la filosofía de los valores de dichos eventos, puede diferir con la auténtica realidad del destino, por lo que en vez de impulsar los verdaderos atributos del país, lo que se estaría haciendo, de hecho, es una ocultación de la imagen real del mismo (sea ésta positiva o negativa). Teniendo presente esta matización, a Brasil se le presenta la oportunidad de variar, establecer o promocionar la imagen de destino. Estos esfuerzos se deberían orientar no sólo a formar la imagen para los años de desarrollo de estos mega eventos sino para proyectarla en un futuro.

La noción de seguridad en el turismo es un concepto variable, de carácter personal y subjetivo, depende de las características socio-demográficas del visitante. Los datos parecen reflejar que la experiencia previa de visita al destino por el visitante internacional es uno de los mejores modos de fortalecer la imagen exterior del país debido al grado de credibilidad e imparcialidad que se transmite. La experiencia personal visita al país contribuye a la creación de una imagen más compleja y singular del destino, ayuda a atenuar los estereotipos negativos y aportar una imagen con componentes singulares y matizados. En la formación de esta imagen más personal hay una menor influencia de los elementos inducidos y autónomos de la formación de la imagen y tienen más peso los elementos orgánicos en los cuales la experiencia y el conocimiento personal adquieren mayor protagonismo. En este ámbito se abre un campo de actuación por parte de las autoridades gubernamentales para la mejora de la gestión de la información con el fin de reducir la incertidumbre del viaje.

\section{Referencias}

Amorín, E., Gándara, J. M., Tarlow, P., \& Korstanje, $\mathrm{M}$.

2012 "Seguridad percibida en la ciudad de Curitiba. Un sondeo exploratorio en residentes locales y turistas". Revista de Análisis Turístico, 14: 1-9.

Baloglu, S., \& Mangaloglu, M.

2001 "Tourism destination images of Turkey, Egypt, Greece, and Italy as perceived by US-based tour operators and travel agents". Tourism Management, 22(1): 1-9.
Barker, M., Page, S. J., \& Meyer, D.

2003 "Urban visitor perceptions of safety during a special event". Journal of Travel Research, 41(4): 355-361.

Borkovec, T. D.

1994 The nature, functions, and origins of worry.

Brás, M., \& Rodrigues, V.

2010 "Turismo e crime: Efeitos da criminalidade na procura turistica". Encontros Científicos-

-Tourism \& Management Studies, 6: 59-68.

Cornelissen, S.

2004 “'It's Africa's turn! The narratives and legitimations surrounding the Moroccan and South African bids for the 2006 and 2010 FIFA finals". Third World Quarterly, 25(7): 1293-1309.

Echtner, C. M., \& Ritchie, J. B.

1993 "The measurement of destination image: An empirical assessment". Journal of Travel Research, 31(4): 3-13.

Frigerio, A., \& Ribeiro, G. L.

2002 Argentinos e brasileiros: Encontros, imagens e estereótipos. Brasil: Vozes Petrópolis.

Gartner, W. C.

1994 "Image formation process". Journal of Travel \& Tourism Marketing, 2(2-3): 191-216.

George, R.

2010 "Visitor perceptions of crime-safety and attitudes towards risk: The case of table mountain national park, Cape Town". Tourism Management, 31(6): 806-815.

Gunn, C. A.

1988 Vacationscape: Designing tourist regions. Van Nostrand Reinhold.

Instituto de Seguridad Pública.

2012 Balanço das incidências criminais e administrativas no estado do Rio de Janeiro. Retrieved 05/24, 2013, from http://www.isp. rj.gov.br/Conteudo.asp ?ident=150.

Jennings, W., \& Lodge, M.

2009 "Governing mega-events: Tools of security risk management for the London 2012 Olympic Games and FIFA 2006 world cup in Germany". Proceedings of the Political Studies Association Conference,

Kim, S. S., \& Morrsion, A. M.

2005 "Change of images of south Korea among foreign tourists after the 2002 FIFA world cup”. Tourism Management, 26(2): 233-247.

Korstanje, M.

2010 "El 11 de septiembre y la teoría de la percepción del riesgo". Pasos, 8(2): 389-402.

Lepp, A., \& Gibson, H.

2003 "Tourist roles, perceived risk and international tourism". Annals of Tourism Research, 30(3): 606-624. 
Llopis Goig, R.

2012 Megaeventos deportivos. perspectivas científicas y estudios de caso. Barcelona: Editorial UOC.

Ministerio de Turismo de Brasil.

2010 Referencial turismo Brasil 2011-2014. Brasilia. Retrieved 05/27, 2013, from http://www. turismo.gov.br/export/sites/default/turismo/o_ minis terio/publicacoes/downloads_publicacoes/Turismo_no_Brasil_2011_-_2014_sem_ margem_corte.pdf.

Ministerio de Turismo de Brasil.

2012 Estudio demanda turística internacional 2005-2011. Retrieved 05/27, 2013, from http://www.dadosefatos.turismo.gov.br/export/ sites/default/ dadosefatos/demanda_turistica/downloads_demanda/Demanda_Turxstica_Internacional_-_Fichas_Sinteses_ -_2005-2011_V2.pdf.

Neirotti, L. D., \& Hilliard, T. W.

2006 "Impact of Olympic spectator safety perception and security concerns on travel decisions". Tourism Review International, 10(4): 269-284.

Payne, M.

2007 Oro olímpico. Madrid: LID Editorial Empresarial.

Radicchi, E.

2012 "Megaeventos deportivos y creación de valor para las economías anfitrionas". In R. Llopis Goig (Ed.), Megaeventos deportivos. perspectivas científicas y estudios de caso (pp. 25-52). Barcelona: Editorial UOC.

Reisinger, Y., \& Mavondo, F.

2005 "Travel anxiety and intentions to travel internationally: Implications of travel risk perception". Journal of Travel Research, 43(3): 212-225.

Ribeiro, G. L.

2002 "Tropicalismo e europeísmo. modos de representar o Brasil e a Argentina”. In A. Frigerio, \& G. L. Ribeiro (Eds.), Argentinos e brasileiros: Encontros, imagens e estereótipos (pp. 1-18). Brasil: Vozes Petrópolis.

Ritchie, J. B.

1984 "Assessing the impact of hallmark events: Conceptual and research issues". Journal of Travel Research, 23(1): 2-11.

Santana, J. D. M., \& Palacio, A. B.

2004 "Cómo influyen las fuentes de información en la imagen percibida de los destinos turísticos". Revista Española de Investigación de Marketing, 8(2): 7-34.

Schroeder, A., Pennington-Gray, L., Kaplanidou, K., \& Zhan, F.

2013 "Destination risk perceptions among US residents for London as the host city of the 2012 summer Olympic Games". Tourism Management, 38: 107-119.
Slevitch, L., \& Sharma, A.

2008 "Management of perceived risk in the context of destination choice". International Journal of Hospitality \& Tourism Administration, 9(1): 85-103.

Soto Navarro, S.

2005 "La influencia de los medios en la percepción social de la delincuencia". Revista Electrónica de Ciencia Penal y Criminología, (7): 9.

Stangeland, P., Díez Ripollés, J. L., \& Durán, M. A. 1998 El blanco más fácil: La delincuencia en las zonas turísticas. Valencia: Tirant lo Blanch.

\section{Notas}

1 MIGRACOM (Observatorio y Grupo de Investigación sobre Migración y Comunicación) Universidad Autónoma Barcelona 2008 Nicolás Lorite y Maria Bade.

2 "Cada día, 16 personas son violadas en Río de Janeiro", El Mundo, 15/05/2013; "Una violación y un vídeo exponen grietas de seguridad en Brasil” Edición digital BBC, 2/04/2013.

Recibido:

$02 / 05 / 2013$

Reenviado:

$15 / 06 / 2013$

Aceptado:

$01 / 07 / 2013$

Sometido a evaluación por pares anónimos 